Mitmachen und gewinnen

\title{
Innovationspreis 2015 - praktikable Problemlösungen für Ärzte
}

— Die Zeit läuft. Noch bis zum 30. November können erfinderische und gut organisierte Ärzte punkten und attraktive Preise gewinnen.

Haben Sie eine innovative Idee, die Sie in Ihrer Praxis umsetzen wollen oder umgesetzt haben? Haben Sie Ihre Praxis gut organisiert, sodass Sie dem Versorgungsdruck standhalten, ohne mehr arbeiten zu müssen? Haben Sie gute Ideen, wie Sie Ihr Team motivieren können? Arbeiten Sie effizient mit moderner Medizintechnik, mit Hard- oder Software? Oder sehen Sie anderweitig in Ihrer Praxisführung innovatives Potenzial? Dann machen Sie mit beim Wettbewerb "Die innovative Arztpraxis 2015".

Nicht nur große Erfindungen, neue Geräte oder Computer-Programme stehen bei der Jury hoch im Kurs. Die kleinen Ideen sind ebenfalls gefragt, die den Praxisalltag vereinfachen und zum Beispiel Patienten helfen, schneller einen Arzttermin zu bekommen. Zeigen Sie Ihren Kollegen, wie Sie es schaffen, die Herausforderungen in der Praxis zu meistern! Sie können mit Ihrer Idee ein Befragungstool HCC Analytics des Beratungsunternehmens HCC BetterCare (Köln) zur Optimierung der Praxis gewinnen. Darüber hinaus gibt es Buchpreise von Springer Medizin.

Der Preis wird 2015 zum fünften Mal ausgeschrieben. Die Bewerbung kann online erfolgen.

Unter www.aerztezeitung.de/innovationspreis finden Sie ein Formular, auf dem Sie Ihre Idee und Umsetzung kurz beschreiben können. Alternativ dazu können Bewerbungsunterlagen per E-Mail an innovation@aerztezeitung.de eingesendet werden. Eine unabhängige Jury entscheidet über die Preisträger. Hauke Gerlof

Der Wettbewerb ist eine Initiative des Biopharmaunternehmens UCB und der Fachverlagsgruppe Springer Medizin. Ihre Daten werden nur zur Ermittlung der Gewinner verwendet und nicht an Dritte weitergeleitet.

\section{Risikomanagement}

\section{Warum viele Praxen in die Risikofalle tappen}

\section{Seit mehr als einem Jahr besteht für Praxen die Pflicht für ein internes Risiko- management. Noch klappt es nicht überall. Dabei können Praxen schnell nachbessern.}

—ür das seit April 2014 geltende interF ne Risikomanagement müssen Praxen mehr bieten als ein Fehlermanagement. Doch daran scheitern viele, weil sie Fehler in der Kommunikation machen, berichtet die TÜV SÜD Akademie.

Dreh- und Angelpunkt eines funktionierenden Risikomanagements ist nach Erfahrung des TÜV SÜD eine offene Fehlerkultur. Sie zeichne sich dadurch aus, dass die Mitarbeiter den Wert von Fehlern als Indikatoren für systematische Risiken erkennen. Fehlermeldungen müssen aber auch von der Praxisleitung eingefordert werden. Helfen können anonyme Meldesysteme. In kleinen Praxen ist so etwas meist schwierig umzusetzen, hier können sich die Teams am Fehlermeldesystem der Universität Frankfurt/ Main jeder-fehler-zaehlt.de beteiligen und zugleich aus Fehlern und BeinaheFehlern anderer Praxen lernen. Insgesamt besteht der Risikomanagementprozess laut TÜV SÜD aus sechs Phasen: Steuerung und Rahmenbedingungen in Praxis und Klinik; Risikoidentifikation; Risikoanalyse; Risikobewertung; Risikobewältigung sowie Risikoüberwachung und Kommunikation.

Spannend sind aber auch die Gründe für häufiges Scheitern: Neben einer mangelhaften Risikopolitik und Fehlerkultur sei dies laut Erfahrungen des TÜV SÜD vor allem eine Praxisleitung, die nicht hinter dem System steht. Problematisch seien auch zu geringe monetäre und personelle Ressourcen. Häufig sind es die strukturellen Probleme, wie zunehmende Komplexität in der Medizin bei dünner werdender Personaldecke, welche die Anfälligkeit für Fehler erhöhen. Zu den Gründen zählen auch eine mangelnde Zielorientierung der Risikopartner in der Praxis, fehlende Messbarkeit der RisikoZielbearbeitung und unzureichende Kommunikation hinsichtlich der Risikoerfassung und -verbesserung.

Schnell nachbessern könnten Praxen bei folgendem Punkt: Oft sind Verantwortlichen nicht klar benannt oder es werden nicht alle Risikobereiche identifiziert und priorisiert. „Es ist sinnvoll einen Mitarbeiter in der Einrichtung als Risikomanager zu benennen und zu qualifizieren“, so der TÜV SÜD. Das kann der Praxisinhaber selbst sein, es wäre aber auch eine Möglichkeit, um einer medizinischen Fachangestellten mehr Kompetenzen zu übertragen. RebekkaHöhl 